\title{
硅胶及其负载酸在糖化学中的应用研究进展
}

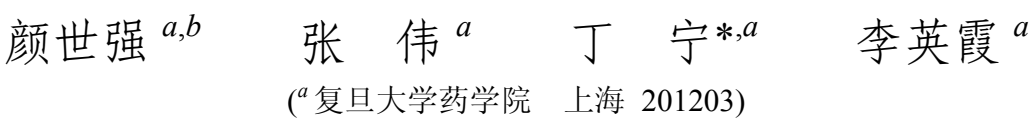 \\ $\left({ }^{b}\right.$ 中国海洋大学医药学院 青岛 266003)
}

\begin{abstract}
摘要＼cjkstart硅胶及其负载酸在有机合成中有着极其广泛的应用. 结合我们的研究工作, 系统综述了硅胶及其负载酸在糖化 学中的应用研究进展. 主要内容包括它们在糖苷化、官能团转化以及水解反应方面的应用.

关键词＼cjkstart硅胶; 固体负载酸; 糖化学; 糖苷化; 官能团转化
\end{abstract}

\section{Application Progress of Silica Gel Supported Acids in Carbohydrate Chemistry}

\author{
Yan, Shiqiang ${ }^{a, b} \quad{\text { Zhang, } \mathrm{Wei}^{a} \quad \text { Ding, Ning }}^{*, a} \quad$ Li, Yingxia ${ }^{a}$ \\ ( ${ }^{a}$ School of Pharmacy, Fudan University, Shanghai 201203) \\ $\left({ }^{b}\right.$ School of Pharmacy, Ocean University of China, Qingdao 266003)
}

\begin{abstract}
Silica gel and its supported acids have been used widely in organic synthesis. Based on our researches, their applications in carbohydrate chemistry are reviewed mainly focusing on the glycosylation, functional group transformation, and hydrolysis applications.

Keywords silica gel; solid supported acids; carbohydrate chemistry; glycosylation; functional group transformation
\end{abstract}

酸性催化剂在现代有机合成, 尤其是工业生产中有 着极为广泛的应用. 据统计每年有超过 $1 \times 10^{8} \mathrm{t}$ 的产品 生产用到酸性催化剂 ${ }^{[1]}$. 在所有的酸性催化剂中, $\mathrm{HClO}_{4}, \mathrm{H}_{2} \mathrm{SO}_{4}, \mathrm{H}_{3} \mathrm{PO}_{4}$ 以及 $\mathrm{HF}$ 的使用最为普遍. 但是由 于其固有的强酸性以及强腐蚀性, 使得它们在储存、使 用以及后处理过程中均存在一定风险, 因而给人们的使 用尤其是工业生产带来诸多不便.

为顺应当今 “绿色化学” 发展要求以及社会可持续 发展需要, 经过化学工作者们的不解努力终于将其成功 负载于固体无机载体制成相应的固体负载酸. 令人欣喜 的是, 制备得到的固体负载酸与其相应的液态酸相比 较, 不仅有效地克服了液态酸存在的诸多缺点, 而且其 催化活性亦有不同程度的提高. 硅胶与其它常用的无机 载体相比具有较大的表面积和更高的反应活性, 因而作 为无机载体的使用最为普遍. 硅胶负载酸通常具有如下 优点: 热力学及动力学稳定性、储存及操作方便性、低 毒性、无腐蚀性、经济实用性、环境耐受性、回收再生
性以及后处理简易性.

多相有机反应与传统的液相反应相比不仅能够有 效地简化反应及后处理操作、优化反应条件, 甚至可以 有效地提高反应收率及增强反应选择性 ${ }^{[2,3]}$. 近年来, 多 相催化剂以其在工业生产及技术开发中的重要性, 正在 受到人们越来越多的重视并发展成为当下研究的热点, 关于它们在有机合成中的重要应用也出现了一些综述 性的文献 ${ }^{[4 \sim 6]}$. 本文重点针对硅胶及其负载酸在糖化学 中的应用做一综述.

\section{1 硅胶及其负载酸在糖苷化中的应用}

糖苷化是一种将糖类化合物的异头碳与其它的糖 单元或分子连接在一起的合成方法. 1893 年, Fischer 糖 苷化的诞生拉开了糖苷化的发展大幕 ${ }^{[7]}$. 自此以后, 特 别是近几十年来, 由于糖缀合物特别是糖蛋白及糖脂的 发现，以及人们对于糖缀合物中的糖链部分可作为蛋白 的抗原或受体认识的不断深入, 大大促进了䓖糖合成方

\footnotetext{
* E-mail: dingning@fudan.edu.cn

Received March 3, 2012; revised May 9, 2012; published online June 1, 2012.

Project supported by the National Natural Science Foundation of China (Nos. 81072525, 21002014).

国家自然科学基金(Nos. 81072525, 21002014)资助项目。
} 
法的研究及发展. 随着新试剂及新合成方法的不断涌 现, 糖化学尤其是糖苷化的发展进入了快车道 ${ }^{[8 \sim 12]}$.

\section{1 硅胶在糖苷化中的应用}

硅胶作为催化剂应用于糖苷化反应的文献报道最 早见诸于 1997 年 ${ }^{[13]}$. 室温条件以苯作为反应溶剂在 3 个质量分数硅胶以及 9 个质量分数分子笁的存在下, 1,2-脱水-3,4,6-三- $O$-新戊酰基- $\alpha$ - $D$-吡喃葡萄糖（1a)与 环已醇发生糖苷化反应, 以 $72 \%$ 的收率得到 $\beta$ 构型化合 物 2a 以及部分开环水解产物 3a (Eq. 1). 在此反应中分 子篮的使用与否对实验结果影响较大, 作者认为其在该 反应过程中扮演了除水剂的角色, 能有效地减少开环水 解产物的生成从而提高相应糖苷化产物的收率.
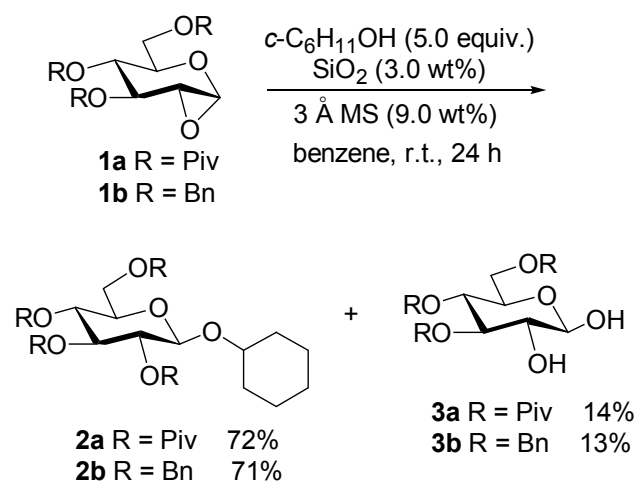

利用该方法作者还成功地合成了几个天然存在的 萜类葡萄糖苷化合物. 化合物 $1 \mathrm{a}$ 与异丙醇、香叶醇、 (一)-紫苏子醇以及酚羟基由四氢吡喃基保护的松柏醇 在硅胶的催化下能够以较好的收率得到相应的糖苷化 产物 4a 4d. 但是当化合物 $1 \mathrm{a}$ 与空间位阻相对较大的 反式及顺式香芹醇反应时收率较低(Scheme 1). 随之脱 除保护基则得到相应的萜类葡萄糖苷天然化合物 5d, 5f 以及 6.

\section{2 硅胶负载酸 $\mathrm{HClO}_{4}-\mathrm{SiO}_{2}$ 在糖苷化中的应用}

关于硅胶负载高氯酸 $\left(\mathrm{HClO}_{4}-\mathrm{SiO}_{2}\right)$ 的文献报道最早 见于 2003 年. Chakraborti 等 ${ }^{[14]}$ 在研究 $\mathrm{HClO}_{4}$ 催化醇羟基 乙酰化时发现: $\mathrm{HClO}_{4}$ 催化对酸不稳定的醇羟基乙酰化 时, 常常伴有重排产物生成. 为避免反应过程中副反应 的发生，他们将 $\mathrm{HClO}_{4}$ 负载于硅胶，从而发明了 $\mathrm{HClO}_{4}-\mathrm{SiO}_{2}$ 这一优良的催化体系.

$\mathrm{HClO}_{4}-\mathrm{SiO}_{2}$ 催化体系的出现, 迅速引起了化学工作 者们的高度关注. Agarwal 课题组 ${ }^{[15]}$ 和 Misra 课题组 ${ }^{[16]}$ 相继报道了 $\mathrm{HClO}_{4}-\mathrm{SiO}_{2}$ 催化糖烯的 Ferrier 重排. 在催 化量的 $\mathrm{HClO}_{4}-\mathrm{SiO}_{2}$ 存在下, 3,4,6-三- $O$-乙酰基-葡萄糖 烯(7)能够与伯醇、仲醇、烯丙醇、苯酚、单糖受体以及 硫醇、硫酚等反应以较高的收率得到相应的 2,3-不饱和 $O / S$-糖苷化产物 8 (Eq. 2). 该催化体系不仅高效、反应

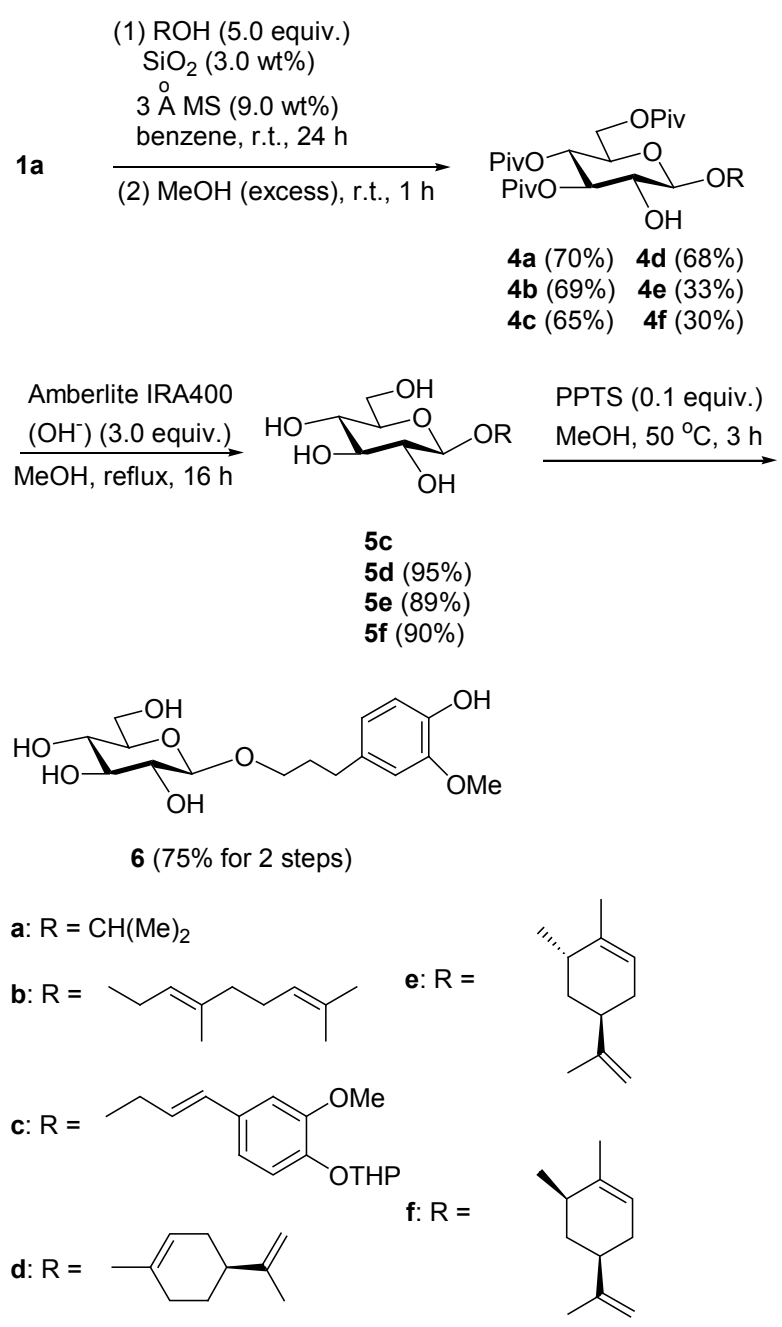

Scheme 1

时间短而且具有很好的 $\alpha$-立体选择性. 文献中作者还将 $\mathrm{HClO}_{4}-\mathrm{SiO}_{2}$ 与 $\mathrm{Yb}(\mathrm{OTf})_{3}, \mathrm{Sc}(\mathrm{OTf})_{3}, \mathrm{CAN}$ 以及 $\mathrm{BF}_{3} \bullet \mathrm{Et}_{2} \mathrm{O}$ 等先前报道的用于 Ferrier 重排的催化试剂进行了比较. 结果显示 $\mathrm{HClO}_{4}-\mathrm{SiO}_{2}$ 作为催化剂无论是在反应时间、 反应收率还是在立体选择性方面均表现出一定的优 势 $[15]$.

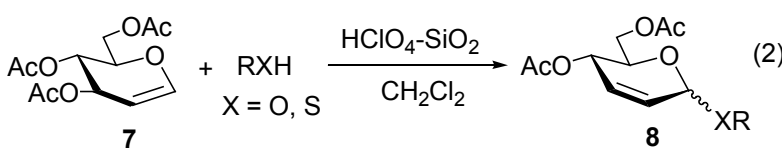

2005 年, Misra 课题组 ${ }^{[17]}$ 详细报道了室温条件下以 乙腈为溶剂, $\mathrm{HClO}_{4}-\mathrm{SiO}_{2}$ 催化糖烯与 $C$-亲核试剂的 Ferrier 重排来制备 2,3-不饱和- $C$-苷类化合物 10 (Eq. 3). 该反应操作简便、反应时间较短、收率较高、 $\alpha: \beta$ 立 体选择性比例为 $1.5 ： 1 \sim 20 ： 1$, 是一种制备糖类 $C$-苷 化合物的不错选择.

$\mathrm{HClO}_{4}-\mathrm{SiO}_{2}$ 同样可以催化 Ferrier 重排来制备 $N$-苷 类化合物. 2008 年, Colinas 等 ${ }^{[18]}$ 报道了 $\mathrm{HClO}_{4}-\mathrm{SiO}_{2}$ 催化 


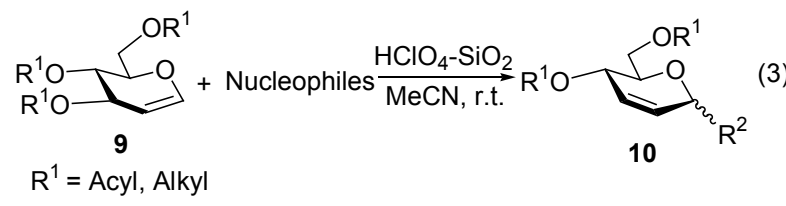

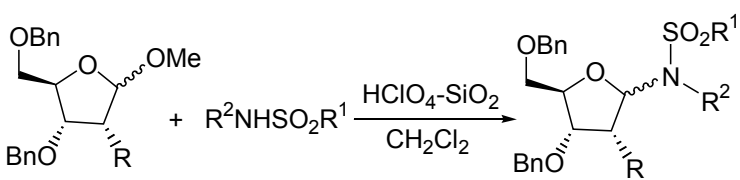

$11 \mathrm{R}=\mathrm{Bn}$ $12 \mathrm{R}=\mathrm{H}$

$13 \mathrm{R}=\mathrm{Bn}$ $14 \mathrm{R}=\mathrm{H}$

甲基苷与磺酰胺反应制备 $N$-磺酰胺类化合物(Eq. 4). 该 反应时间较短、后处理简单且收率较高, 不失为制备 $N$ 磺酰胺类化合物的明智选择.

关于 $\mathrm{HClO}_{4}-\mathrm{SiO}_{2}$ 作为催化剂应用于寡糖合成的文 献报道最早见诸于 2005 年. Mukhopadhyay 等 ${ }^{[19]}$ 使用 $\mathrm{NIS} / \mathrm{HClO}_{4}-\mathrm{SiO}_{2}$ 替代 NIS/TfOH 体系来催化全乙酰化硫 苷的糖苷化, 以 $74 \% \sim 87 \%$ 的收率得到相应的 1,2-反式 糖苷化产物. 利用该法他们成功的以一步 $72 \%$ 的收率实 现了凝血素伴刀豆球蛋白 $\mathrm{A}$ 核心甘露三糖 17 的合成 (Eq. 5).

2007 年, Mukherjee 等 ${ }^{[20]}$ 使用 $\mathrm{NIS} / \mathrm{HClO}_{4}-\mathrm{SiO}_{2}$ 体系 催化全乙酰化单糖或者双糖乙硫苷与 1,$2 ; 3,4$-二- $O$-异亚 丙基- $\alpha-D$-吡喃半乳糖的糖苷化, 所得双糖或三糖化合 物在醇的存在下以 $\mathrm{HClO}_{4}-\mathrm{SiO}_{2}$ 为催化剂成功实现了半 乳糖由六元吡喃型到五元呋喃型的异构化(Scheme 2). 有意思的是当作者用 $70 \% \mathrm{HClO}_{4}$ 替代 $\mathrm{HClO}_{4}-\mathrm{SiO}_{2}$ 时发 现异构化将不会发生.

Mukhopadhyay 课题组 ${ }^{[21]}$ 同样应用 $\mathrm{HClO}_{4}-\mathrm{SiO}_{2}$ 作为 催化剂成功实现了全乙酰化三氯乙酰亚胺酯给体的糖 苷化. 令人欣喜的是作者还采用 “一锅法” 合成策略分 别以 $62 \%$ 和 $59 \%$ 的收率成功合成了 $\mathrm{Le}^{\mathrm{x}} 25$ 以及 $\mathrm{Le}^{\mathrm{a}} 26$ 三糖衍生物(Scheme 3).

为进一步简化实验操作, 在该篇文献中作者还介绍 了 “on-column” 合成法. 运用该合成方法作者以较好的 收率成功实现了全乙酰化三氯乙酰亚氨酯给体与氨基 酸的糖苷化来制备糖肽类化合物 ${ }^{[21]}$. 使用价廉易得、低 毒易操作的 $\mathrm{HClO}_{4}-\mathrm{SiO}_{2}$ 替代 TMSOTf 作为催化剂, 不 仅有利于缩短反应时间、提高某些反应的收率而且更加 符合 “绿色化学”发展的需要.

$\mathrm{Du}$ 等 ${ }^{[22]}$ 系统研究了 $\mathrm{HClO}_{4}-\mathrm{SiO}_{2}$ 催化三氯乙酰亚胺 酯给体的糖苷化. 实验结果表明糖类的羟基常用保护基 如异亚丙基、苯亚甲基、乙酰基、苯甲酰基、烯丙基、 苠基、三苯甲基、叔丁基二甲基以及其它官能团如内酯、 醛基、叠氮基、硫苷等在此糖苷化过程中均不受影响. 在该文献中作者还指出, 某些使用 TMSOTf催化无法完
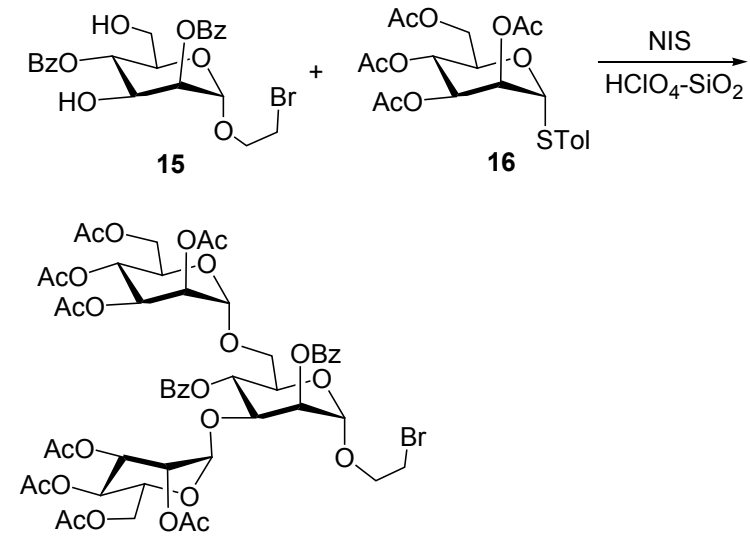

17
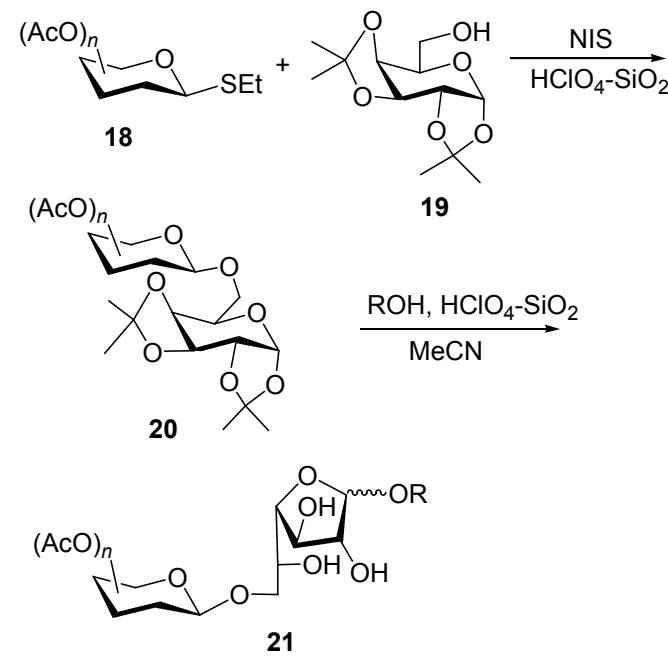

Scheme 2

成的反应使用 $\mathrm{HClO}_{4}-\mathrm{SiO}_{2}$ 催化可顺利实现. 例如作者 利用此方法几乎以定量的收率得到了阿维菌素 B1a 衍 生物 29, 而该反应采用 TMSOTf 或者 $\mathrm{BF}_{3} \cdot \mathrm{Et}_{2} \mathrm{O}$ 催化收 率较低(Eq. 6).

2010 年, Ludek 等 ${ }^{[23]}$ 经过深入研究后指出: $\mathrm{HClO}_{4}-\mathrm{SiO}_{2}$ 在催化没有邻基参与基团存在三氯乙酰亚 氨酯给体的糖苷化过程中较之常用的 TMSOTf 能够明 显地提高产物的 $\alpha$ 立体选择性. 例如在三氯乙酰亚氨酯 给体 30 与受体 31 的糖苷化过程中, 采用传统的 TMSOTf作为催化剂其 $\alpha: \beta$ 立体选择性的比例仅为 5.3. 保持其余反应条件不变，仅将催化剂更换为 $\mathrm{HClO}_{4}-\mathrm{SiO}_{2}$, 则其 $\alpha: \beta$ 立体选择性大大提高(表 1). 上 述结果显示出 $\mathrm{HClO}_{4}-\mathrm{SiO}_{2}$ 作为催化剂在催化无邻基参 与基团存在的三氯乙酰亚氨酯给体的糖苷化过程中具 有明显的优势.

Agnihotri 等 ${ }^{[24]}$ 充分利用 $\mathrm{HClO}_{4}-\mathrm{SiO}_{2}$ 这一催化剂, 经 13 步操作成功合成 Escherichia coli O83:K24:H31 O抗原的三糖甲基苷类似物, 其中 5 步操作应用了 $\mathrm{HClO}_{4}$ - 


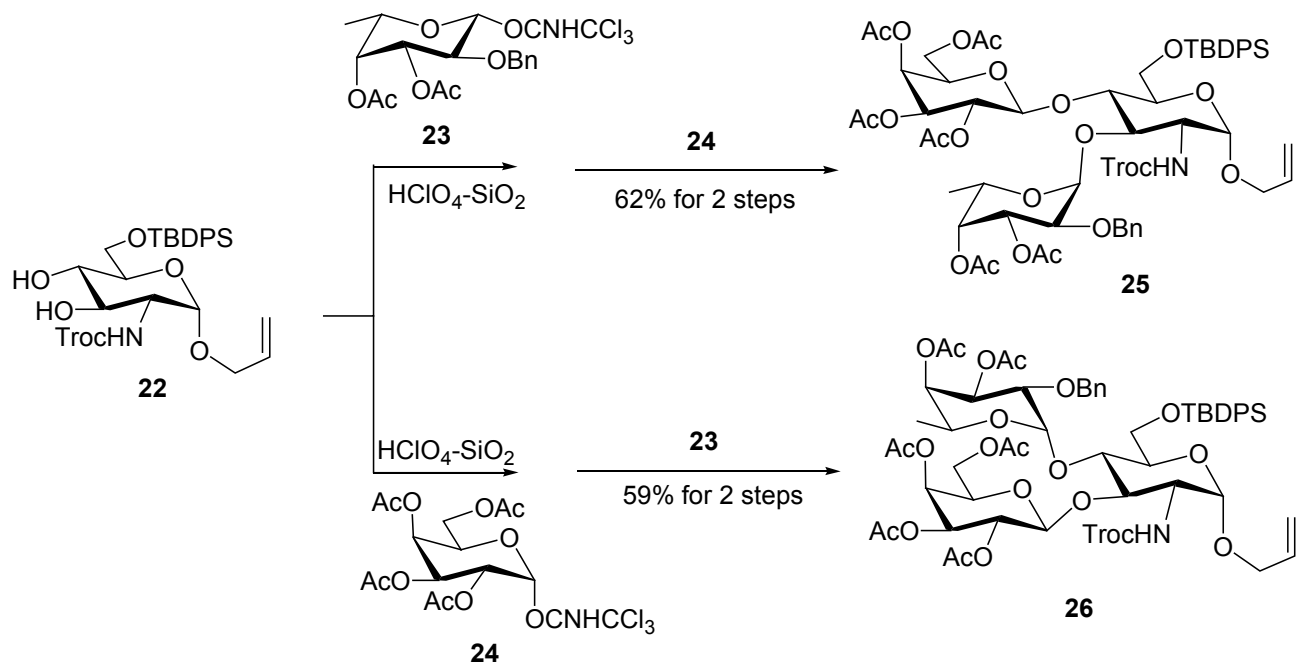

Scheme 3

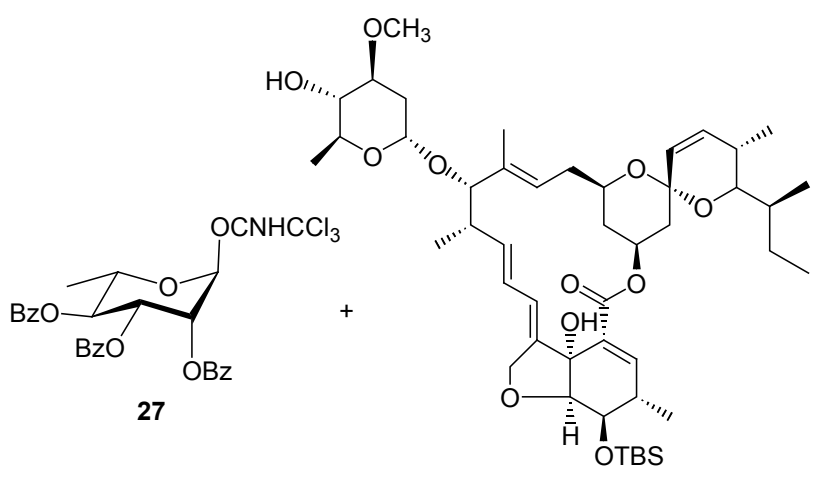

28

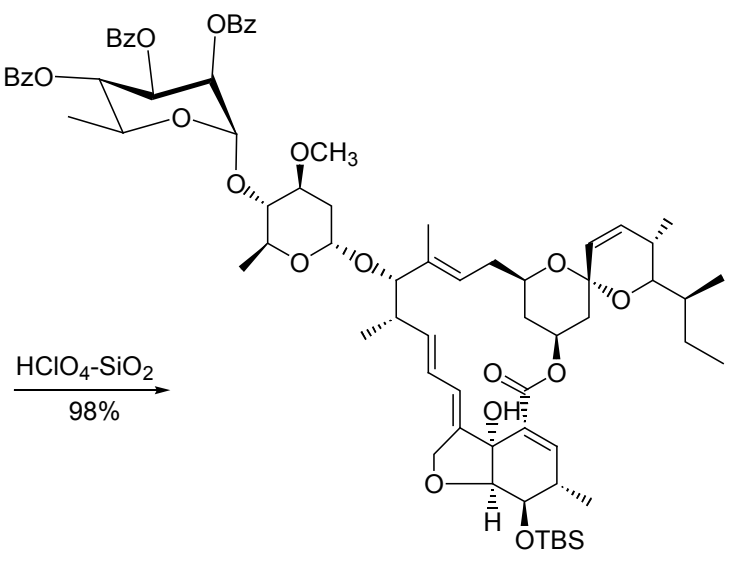

29

$\mathrm{SiO}_{2}$ 催化体系. $\mathrm{HClO}_{4}-\mathrm{SiO}_{2}$ 的使用大大简化了实验操作.

我们课题组 ${ }^{[25]}$ 最近报道了 $\mathrm{HClO}_{4}-\mathrm{SiO}_{2}$ 作为催化剂 催化分子内糖苷化来制备 1,6-脱水内醚糖(Eq. 7). 以乙 腈为反应溶剂, $70{ }^{\circ} \mathrm{C}$ 下, 6-OH 由 TBDPS 保护的对甲氧 基苯基糖苷在 0.2 equiv. 的 $\mathrm{HClO}_{4}-\mathrm{SiO}_{2}$ 的催化下发生分 子内的自身糖苷化得到相应的 1,6-脱水内醚糖. 该法反 应时间很短(仅为几分钟)、收率较高、后处理简单, 为 1,6-脱水内醚糖的合成提供了一种不错的选择.
表 1 催化剂对糖苷化立体构型的影响

Table 1 Influence of catalyst on stereoselectivity

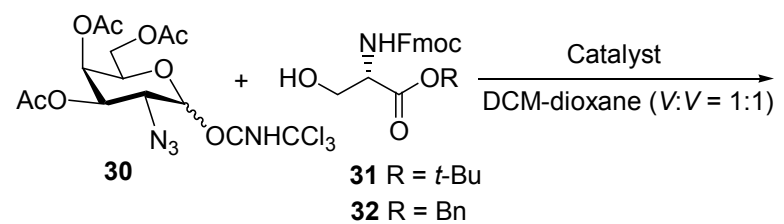

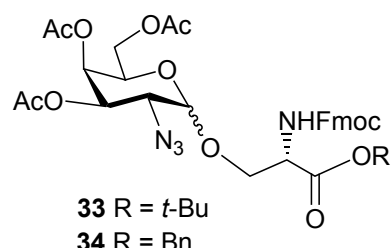

\begin{tabular}{cclccc}
\hline Entry & Acceptor & Catalyst & Temperature Yield/\% & $\alpha: \beta$ \\
\hline 1 & $\mathbf{3 1}$ & $\mathrm{TMSOTf}$ & r.t. & 86 & 5.3 \\
2 & $\mathbf{3 1}$ & $\mathrm{HClO}_{4}-\mathrm{SiO}_{2}$ & r.t. & 42 & 24.0 \\
3 & $\mathbf{3 2}$ & $\mathrm{TMSOTf}$ & r.t. & 98 & 3.7 \\
4 & $\mathbf{3 2}$ & $\mathrm{HClO}_{4}-\mathrm{SiO}_{2}$ & r.t. & 95 & 12.0 \\
\hline
\end{tabular}

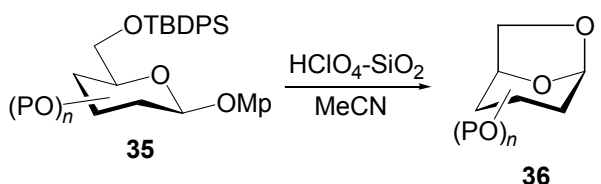

\section{3 硅胶负载酸 $\mathrm{H}_{2} \mathrm{SO}_{4}-\mathrm{SiO}_{2}$ 在糖苷化中的应用}

由于 $\mathrm{HClO}_{4}-\mathrm{SiO}_{2}$ 具有一定的潜在爆炸性, 随后人 们发展了安全性能更高的硅胶负载硫酸 $\left(\mathrm{H}_{2} \mathrm{SO}_{4}-\mathrm{SiO}_{2}\right)$ 予 以替代.

$\mathrm{H}_{2} \mathrm{SO}_{4}-\mathrm{SiO}_{2}$ 被成功应用于 Fisher 糖苷化. 2007 年 Roy 等 ${ }^{[26]}$ 将不保护的单糖(例如 $D$-葡萄糖、 $D$-半乳糖、 $D$-甘露糖、 $L$ - 鼠李糖、 $L$-岩藻糖、 $D$-氨基葡萄糖、 $D$-麦 芽糖)与 5 equiv. 的各种醇在 $\mathrm{H}_{2} \mathrm{SO}_{4}-\mathrm{SiO}_{2}$ 的催化下于 65 
${ }^{\circ} \mathrm{C}$ 摚拌反应 $2 \sim 8 \mathrm{~h}$, 以 $69 \% \sim 83 \%$ 的收率得到相应的 Fischer 糖苷化产物. 该法与传统的 Fischer 糖苷化相比 不仅大大减少了相应的醇用量、缩短了反应时间、降低 了反应温度, 而且在很大程度上提高了收率. Shaikh 等 ${ }^{[27]}$ 于 2010 年同样报道了超声辅助下的 $\mathrm{H}_{2} \mathrm{SO}_{4}-\mathrm{SiO}_{2}$ 催 化 Fisher 糖苷化.

2010 年 Richel 等 ${ }^{[28]}$ 报道了微波辅助 $\mathrm{H}_{2} \mathrm{SO}_{4}-\mathrm{SiG}_{60}$ 催 化 $D$-葡萄糖醛酸与 5 equiv. 的醇反应制备相应的 6,3-内 酯呋喃葡萄糖糖苷化产物 38 (Eq. 8).

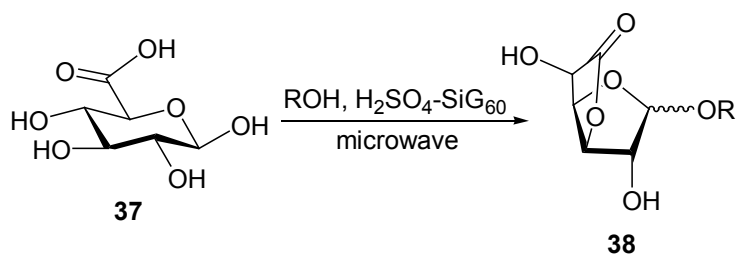

$\mathrm{H}_{2} \mathrm{SO}_{4}-\mathrm{SiO}_{2}$ 同样可用于催化 Ferrier 重排. 2010 年, Zhou 等 ${ }^{[29]}$ 报道了 $\mathrm{H}_{2} \mathrm{SO}_{4}-\mathrm{SiO}_{2}$ 催化 3,4,6-三- $O$-乙酰基葡萄糖烯(7)与醇的 Ferrier 重排来制备相应的 2,3-不饱 和- $O$-糖苷化产物.

文献报道的关于 $\mathrm{H}_{2} \mathrm{SO}_{4}-\mathrm{SiO}_{2}$ 催化三氯乙酰亚胺酯 给体的糖苷化以及 $\mathrm{NIS} / \mathrm{H}_{2} \mathrm{SO}_{4}-\mathrm{SiO}_{2}$ 催化硫苷给体的糖 苷化反应 ${ }^{[30 ~ 36]}$ 几乎全部来自印度的 Mukhopadhyay 研 究小组. 2007 年以来, 他们使用这一催化体系合成了一 系列天然产物中的相应糖苷部分. 例如, 2008 年, 他 们 ${ }^{[32]}$ 采用 $\mathrm{H}_{2} \mathrm{SO}_{4}-\mathrm{SiO}_{2}$ 催化三氯乙酰亚胺酯给体的糖苷 化以及 $\mathrm{NIS} / \mathrm{H}_{2} \mathrm{SO}_{4}-\mathrm{SiO}_{2}$ 催化硫苷给体的糖苷化反应成 功合成了从 Maesa balansae 中分离得到的具有抗利什曼 原虫活性的三萜㿝苷的五糖片段 39 (Scheme 4). 在大量 的实验基础上他们明确指出采用 $\mathrm{H}_{2} \mathrm{SO}_{4}-\mathrm{SiO}_{2}$ 催化体系 相比传统使用的 Lewis 酸, 譬如 TMSOTf, TfOH 其实验 结果相当甚至更优. 更为重要的是 $\mathrm{H}_{2} \mathrm{SO}_{4}-\mathrm{SiO}_{2}$ 为固体 粉末可以准确方便的称量加入, 且其对无水条件的要求 相对要缓和得多.
2007 年, $\mathrm{Wu}$ 等 ${ }^{[37}$ 报道了 $\mathrm{H}_{2} \mathrm{SO}_{4}-\mathrm{SiO}_{2}$ 催化葡萄糖与 烷基醇的直接糖苷化来制备烷基糖苷非离子表面活性 剂 40. 催化剂的用量仅为 $1 \%$, 在 $110{ }^{\circ} \mathrm{C}$ 下反应 $1.5 \mathrm{~h}$, 葡萄糖的转化率几乎为定量(Eq. 9).
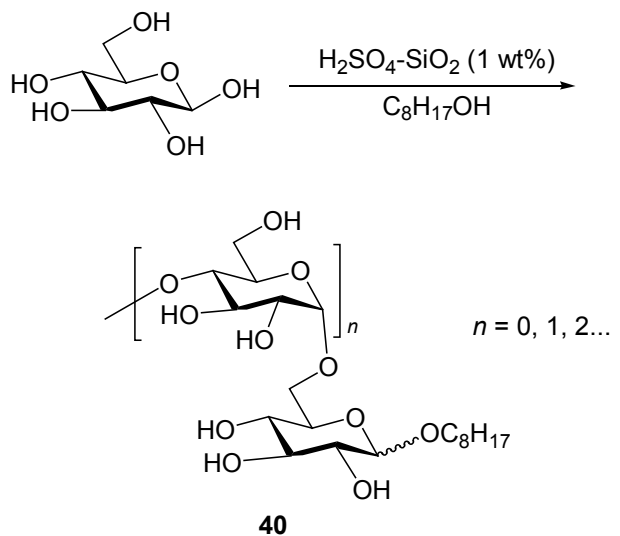

\section{4 其它硅胶负载酸在糖苷化中的应用}

在硅胶负载酸中 $\mathrm{HClO}_{4}-\mathrm{SiO}_{2}$ 及 $\mathrm{H}_{2} \mathrm{SO}_{4}-\mathrm{SiO}_{2}$ 的出现 较早, 相应的它们在有机合成中的应用也最为广泛. 但 是随着科学研究以及工业生产的发展需要，一些新的硅 胶负载酸如硅胶负载三氟甲烷磺酸 $\left(\mathrm{TfOH}-\mathrm{SiO}_{2}\right)$, 硅胶 负载磷钼酸 $\left(\mathrm{PMA}-\mathrm{SiO}_{2}\right)$, 硅胶负载氟硼酸 $\left(\mathrm{HBF}_{4}-\mathrm{SiO}_{2}\right)$, 甚至硅胶负载亚硫酸氢钠 $\left(\mathrm{NaHSO}_{4}-\mathrm{SiO}_{2}\right)$ 等不断出现.

2009 年, Rodriguez 等 ${ }^{[38}$ 报道了在 $\mathrm{HBF}_{4}-\mathrm{SiO}_{2}$ 的催化 下 3,4,6-三- $O$-乙酰基-葡萄糖(半乳糖)烯能够与醇以及 磺酰胺反应以较高的收率得到相应的 2,3-不饱和- $O / N$ 糖苷化产物. 该催化体系不仅高效，反应时间短而且具 有很好的 $\alpha$-立体选择性. $\mathrm{NaHSO}_{4}-\mathrm{SiO}_{2}$ 同样可被用来催 化糖稀的 Ferrier 重排 ${ }^{[39]}$.

\section{2 硅胶负载酸在糖化学官能团转化中的应用}

众所周知，糖类为多羟基醛或者多羟基酮类化合 物, 分子中多个羟基的存在为其化学选择性及立体选择

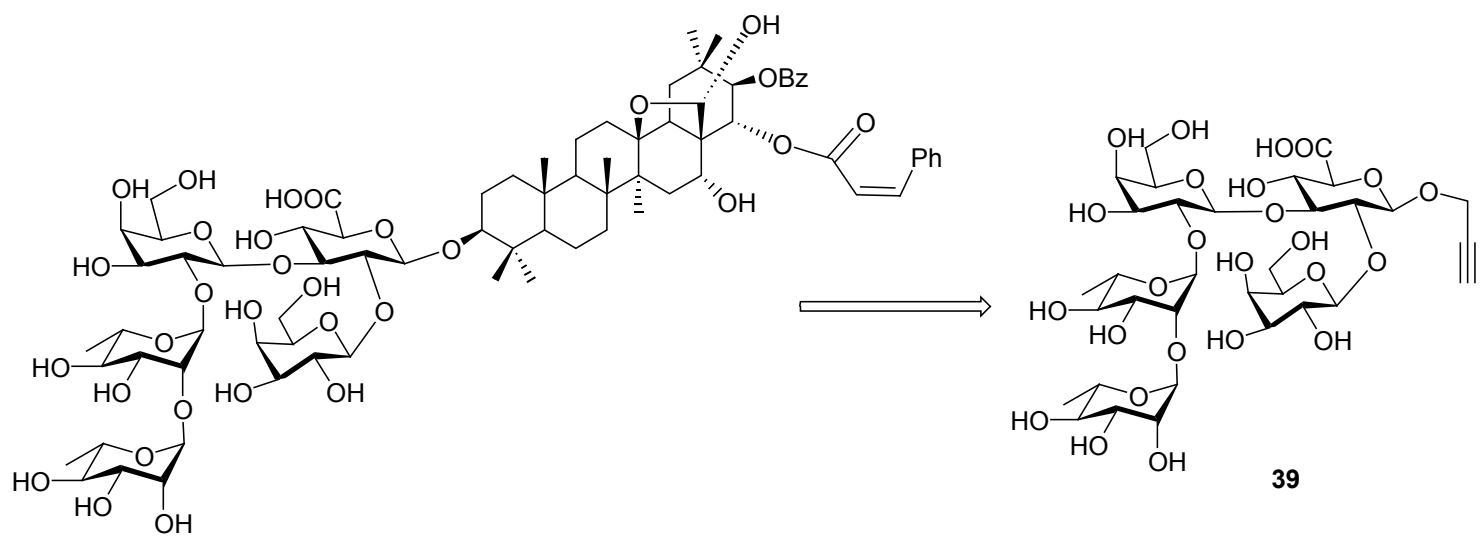

Scheme 4 
性反应带来了诸多困难. 基于此, 对糖类化合物的羟基 进行选择性保护、脱保护以及不同官能团之间的相互转 化就显得尤为重要. 长久以来, 糖类化合物羟基的选择 性保护以及脱保护一直是化学工作者们研究的重要领 域之一, 新的反应试剂、新的反应催化剂以及新的反应 合成策略等不断涌现. 尤其是近年来, 硅胶负载酸在糖 类化合物的着基保护、脱保护以及不同官能团之间的相 互转化方面发展迅速, 出现了一些新方法、新策略.

\section{1 硅胶负载酸 $\mathrm{HClO}_{4}-\mathrm{SiO}_{2}$ 在糖化学官能团转化中的 应用}

2005 年, Misra 等 ${ }^{[40]}$ 报道了催化量的 $\mathrm{HClO}_{4}-\mathrm{SiO}_{2}$ 能 够成功实现不保护或端基保护的单糖、双糖以及三糖裸 露羟基的全乙酰化. 在 $\mathrm{HClO}_{4}-\mathrm{SiO}_{2}$ 的催化下, 使用化学 计量的乙酸䣶即可在非常短的时间内 $(5 \sim 10 \mathrm{~min})$ 以很 高的收率 $(>90 \%)$ 得到相应的乙酰化产物. 在此反应条 件下, 糖类化合物常用的端基保护基均不受影响. 该反 应不需要任何溶剂, 避免了具有难闻气味且具毒性的吡 啶作为常用溶剂的使用, 与传统的羟基乙酰化反应相比 较具有诸多明显优势.

2005 年, Mukhopadhyay 等 ${ }^{[41]}$ 在同一篇文献中报道 了将 $\mathrm{HClO}_{4}-\mathrm{SiO}_{2}$ 应用于端基保护单糖邻二羟基的异亚 丙基化以及苯亚甲基化. 在催化量 $\mathrm{HClO}_{4}-\mathrm{SiO}_{2}$ 的存在 下于无水乙腈中端基保护单糖与 1 equiv.的 2,2-二甲氧 基丙烷反应即可得到其相应的异亚丙基产物. 待反应完 全后，向反应体系中加入乙酸䣶或氯乙酸䣶即可实现剩 余羟基的乙酰化或氯乙酰化, 该方法提供了一种 “一锅 法” 制备异亚丙基化-酰化端基保护的糖类化合物的简 便方法.

同理，端基保护的葡萄糖、半乳糖在 $\mathrm{HClO}_{4}-\mathrm{SiO}_{2}$ 的存在下于无水乙腈中与 1 equiv.的苯甲醛二甲醚反应 得到相应 4,6-苯亚甲基产物，收率为 72\% 94\% ${ }^{[41]}$ 。需 要指出的是甘露糖在无水乙腈中当加入 1 equiv. 的苯甲 醛二甲醚时反应不完全, 当苯甲醛二甲醚增加到 2 equiv. 时以 93\%的收率得到双苯亚甲基化产物 42; 将溶 剂由无水乙腈改换为无水 DMF 后, 能够以 $73 \%$ 的收率 得到相应 4,6-苯亚甲基产物 $\mathbf{4 3}$ (Scheme 5).

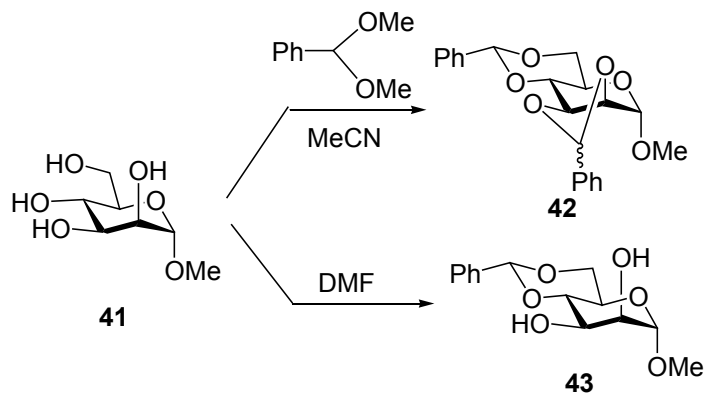

Scheme 5
$\mathrm{HClO}_{4}-\mathrm{SiO}_{2}$ 应用于脱除酸不稳定的三苯甲基亦有 文献报道. 以甲醇为反应溶剂, 室温条件下催化量的 $\mathrm{HClO}_{4}-\mathrm{SiO}_{2}$ 于 $2 \sim 3 \mathrm{~h}$ 内即可选择性的将其脱除 ${ }^{[42]}$. 该 法后处理简单、收率较高, 但对反应溶剂的要求严格, 乙腈会延缓反应进程，而选用二氯甲烷则反应根本不会 发生. 同样的反应条件下末端的异亚丙基亦可被选择性 脱除 ${ }^{[23]}$. 令人高兴的是在该反应条件下, 对酸不稳定的 保护基如叔丁基二甲基硅基，对甲氧基苄基等均不受影 响.

$\mathrm{HClO}_{4}-\mathrm{SiO}_{2}$ 同样可以用于脱除苯亚甲基. 2006 年, Misra 等 ${ }^{[43]}$ 报道了以乙腈为反应溶剂, 催化量的 $\mathrm{HClO}_{4}$ $\mathrm{SiO}_{2}$ 可以迅速脱除单糖或寡糖上面的苯亚甲基得到相 应的邻二醇化合物．同样的反应条件下，乙酸酐的存在 不仅能够加速反应的进行，而且可以同时将得到的羟基 乙酰化，即实现官能团的相互转化(Eq. 10).

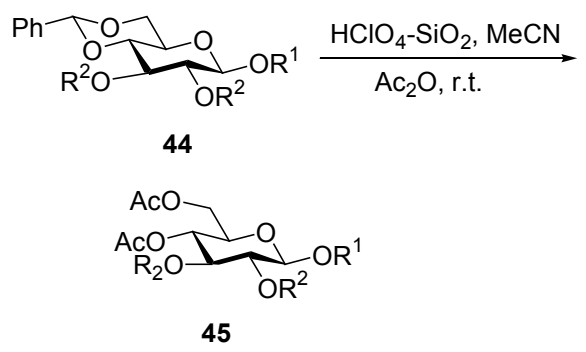

不同官能团之间的相互转化在有机合成化学中有 着非常重要的应用. 2006 年, Agnihotri 等 ${ }^{[44]}$ 报道了在乙 酸䣶的存在下催化量的 $\mathrm{HClO}_{4}-\mathrm{SiO}_{2}$ 可将单糖或者多糖 的各种硫苷转化为其相应的 1-O-乙酰基化合物(Eq. 11). 该反应虽然没有其逆反应在寡糖合成中的应用价值高, 但它为我们在寡糖合成中对于反应条件的选择提供了 有益参考.

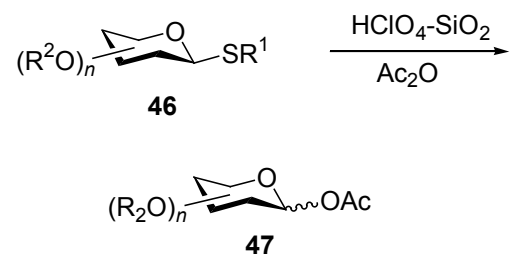

将邻二羟基的异亚丙基保护基一步转化为双乙酰 基保护基, 在有机合成化学尤其是糖化学葟糖合成过程 中有着非常重要的应用. 2009 年, Liu 等 ${ }^{[45}$ 报道了采用 $\mathrm{HClO}_{4}-\mathrm{SiO}_{2}$ 作为催化剂可成功将末端的异亚丙基实现 这一转化，但呋喃糖的端基异亚丙基在该反应条件下得 到相应的乙酰基-(2-乙酰氧基异亚丙基)- $\beta$ - $D$-呋喃糖类 化合物(Scheme 6). 在此需要指出的是, 如果端基异亚 丙基保护的呋喃糖类化合物中含有叔丁基二甲基硅基 保护基, 则相应的羟基亦能够被乙酰化 ${ }^{[46]}$. 即实现了硅 醚保护基到酯基保护基的相互转化. 


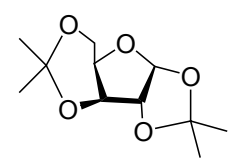

48

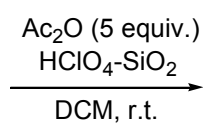

$$
\text { (n) }
$$

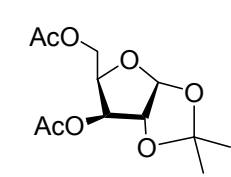

49

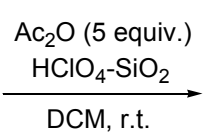

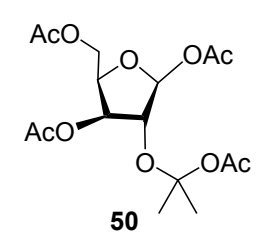

Scheme 6
2.2 硅胶负载酸 $\mathrm{H}_{2} \mathrm{SO}_{4}-\mathrm{SiO}_{2}$ 在糖化学官能团转化中 的应用

$\mathrm{H}_{2} \mathrm{SO}_{4}-\mathrm{SiO}_{2}$ 同样可以催化不保护单糖的全乙酰化. 该法同样无需溶剂、乙酸䣶的用量为化学计量, 与 $\mathrm{HClO}_{4}-\mathrm{SiO}_{2}$ 相比较反应时间稍长 ${ }^{[47]}$.

2006 年, Mukhopadhyay ${ }^{[48]}$ 使用安全性更高的 $\mathrm{H}_{2} \mathrm{SO}_{4}-\mathrm{SiO}_{2}$ 代替 $\mathrm{HClO}_{4}-\mathrm{SiO}_{2}$ 用于端基保护单糖邻二羟 基的异亚丙基化以及芐基化. 以无水乙腈为溶剂, 在 $\mathrm{H}_{2} \mathrm{SO}_{4}-\mathrm{SiO}_{2}$ 的催化下端基保护的葡萄糖、半乳糖以及氨 基葡萄糖与 1 equiv. 的芳基取代苯甲醛二甲醚(例如对甲 氧基苯甲醛二甲醚、对硝基苯甲醛二甲醚、间氯苯甲醛 二甲醚)反应得到相应 4,6-苯亚甲基产物, 其效果同样相 当不错. 待反应完全后, 向反应体系中加入乙酸酮同样 可实现剩余羟基的乙酰化.

稍后 Rajput 等 ${ }^{[49]}$ 报道了 $\mathrm{H}_{2} \mathrm{SO}_{4}-\mathrm{SiO}_{2}$ 催化还原糖的 异亚丙基化: $D$-葡萄糖、 $D$-半乳糖、 $D$-甘露糖、 $L$ - 鼠李 唐、 $L$-阿拉伯糖、 $D$-木糖、 $L$-山梨糖在 $\mathrm{H}_{2} \mathrm{SO}_{4}-\mathrm{SiO}_{2}$ 的催 化下, 于无水丙酮中加热回流能以非常好的收率得到它 们相应的热力学稳定的单异亚丙基或双异亚丙基化产 物(表 2).

2006 年, Mukhopadhyay 等 ${ }^{[00]}$ 应用 $\mathrm{H}_{2} \mathrm{SO}_{4}-\mathrm{SiO}_{2}$ 同样 成功地将二- $O$ - 异亚丙基的 $D$-呋喃葡萄糖、 $D$-呋喃果糖、 $L$-呋喃山梨糖衍生物的末端异亚丙基选择性脱除得到 相应的单异亚丙基产物. 该反应溶剂的影响同样较大, 但比较采用 $\mathrm{HClO}_{4}-\mathrm{SiO}_{2}$ 而言反应时间相对降低, 一般 在 $1 \mathrm{~h}$ 之内完成. 在该反应中酸不稳定的对甲氧基苠基、 叔丁基二甲基硅基等基团均不受影响.

为进一步简化实验操作, 最近 Mukhopadhyay 等 ${ }^{[51]}$ 报道了 “on-column” 法应用 $\mathrm{H}_{2} \mathrm{SO}_{4}-\mathrm{SiO}_{2}$ 脱除苯亚甲基、 三苯甲基、叔丁基二甲基硅基以及选择性脱除末端异亚 丙基.

\section{3 其它硅胶负载酸在糖化学官能团转化中的应用}

最近, 我们课题组 ${ }^{[52]}$ 又报道了 $\mathrm{TfOH}-\mathrm{SiO}_{2}$ 可以选择 性地脱除糖类衍生物 6-OH 的叔丁基二苯基硅基保护基
表 $2 \mathrm{H}_{2} \mathrm{SO}_{4}-\mathrm{SiO}_{2}$ 催化还原糖的异亚丙基化

Table 2 Synthesis of $O$-isopropylidene derivatives from free sugars using $\mathrm{H}_{2} \mathrm{SO}_{4}-\mathrm{SiO}_{2}$

Substrate

以及㿝苷类化合物仲羟基的叔丁基二苯基硅基保护基. 以乙腈为反应溶剂, $50{ }^{\circ} \mathrm{C}$ 下 0.1 equiv. 的 $\mathrm{TfOH}-\mathrm{SiO}_{2}$ 在 $0.5 \sim 3 \mathrm{~h}$ 内可以选择性的脱除硅醚保护基, 糖化学中常 用的其它保护基在此反应条件下不受影响.

其它的硅胶负载酸如 $\mathrm{PMA}-\mathrm{SiO}_{2}{ }^{[53 \sim 55]}, \mathrm{NaHSO}_{4}$ $\mathrm{SiO}_{2}{ }^{[56}$-58]等在糖化学中羟基保护以及脱保护方面的应 用亦有相关的文献报道. 譬如: 它们可以用来脱除叔丁 基二苯基硅基 ${ }^{[53]}$ 、三苯甲基 ${ }^{[56]}$ 、苯亚甲基 ${ }^{[54,57]}$ 、末端异 亚丙基 ${ }^{[55,58]}$ 等. 其作用完全等同于我们前面所述的 $\mathrm{HClO}_{4}-\mathrm{SiO}_{2}$ 以及 $\mathrm{H}_{2} \mathrm{SO}_{4}-\mathrm{SiO}_{2}$, 只不过其酸性要弱于以上 两者.

\section{3 硅胶负载酸在糖化学水解反应中的应用}

$\mathrm{HClO}_{4}-\mathrm{SiO}_{2}$ 可以选择性地脱除异头位乙酰基: 以乙 
腈为反应溶剂, $70{ }^{\circ} \mathrm{C}$ 下催化量的 $\mathrm{HClO}_{4}-\mathrm{SiO}_{2}$ 即可选择 性地将全乙酰化单糖或寡糖的异头位乙酰基顺利脱除 得其相应的半缩醛(Eq. 12) ${ }^{[59]}$. 该反应的溶剂无需无水 处理, 水汽的存在对反应时间以及收率均无影响. 但溶 剂对反应的影响较大, 当反应选用二氯甲烷、三氯甲烷 或者四氢呋喃作溶剂时反应不发生或仅以很低的收率 得到相应的半缩醛. 另外, 该法同样可以选择性地脱除 异头位的苯甲酰基.

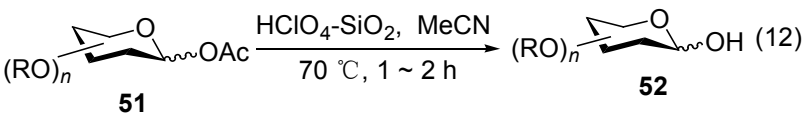

2006 年, Mukhopadhyay 等应用 $\mathrm{NIS} / \mathrm{H}_{2} \mathrm{SO}_{4}-\mathrm{SiO}_{2}$ 体 系实现了硫苷的水解得到其相应的半缩醛. 在 $V(\mathrm{DCM}): V\left(\mathrm{H}_{2} \mathrm{O}\right)=10: 1$ 的混合体系中, 在 1.2 equiv. $\mathrm{NIS}$ 以及催化量 $\mathrm{H}_{2} \mathrm{SO}_{4}-\mathrm{SiO}_{2}$ 的存在下, 单糖、双糖以及 三糖的烷基或芳基硫苷在 30 45 min 内可成功实现水 解, 以 $75 \% \sim 95 \%$ 的收率得到相应的半缩醛产物. 反应 过程中酸不稳定保护基如苯亚甲基、异亚丙基、对甲氧 基芐基、氯乙酰基以及叔丁基二甲基硅基等均不受影 响 ${ }^{[60]}$.

\section{4 结语与展望}

综述了硅胶及其负载酸在糖化学中的应用, 重点介 绍了 $\mathrm{HClO}_{4}-\mathrm{SiO}_{2}$ 以及 $\mathrm{H}_{2} \mathrm{SO}_{4}-\mathrm{SiO}_{2}$ 在糖苷化、官能团转 化以及水解反应等方面的应用. 实践证明: 硅胶负载酸 是对其相应的液态酸的成功改造. 硅胶负载酸以其所具 有的优良特性和对环境的友好性正在受到化学工作者 们越来越广泛的关注, 它们在现代有机合成化学中正在 扮演着越来越重要的角色, 我们坚信随着研究的不断深 入, 新的硅胶负载酸必将会出现, 它们在有机化学尤其 是糖化学中的新应用必将进一步丰富.

\section{References}

[1] Corma, A. Curr. Opin. Solid State Mater. Sci. 1997, (2), p. 63.

[2] Sikdar, S. K.; Howell, S. G. J. Cleaner Prod. 1998, 6, 253.

[3] Varma, R. S. Green Chem. 1999, 1, 43.

[4] Baghernejad, B. Mini-Rev. Org. Chem. 2011, 8, 91

[5] Salehi, P.; Zolfigol, M. A.; Shirinic, F.; Baghbanzadeh, M. Curr. Org. Chem. 2006, 10, 2171.

[6] Rauter, A. P.; Xavier, N. M.; Lucas, S. D.; Santos, M. Adv. Carbohydr. Chem. Biochem. 2010, 63, 29.

[7] Fischer, E. Ber. Deutsch. Chem. Ges. 1893, 26, 2400.

[8] Toshima, K.; Tatsuta, K. Chem. Rev. 1993, 93, 1503

[9] Hanessian, S.; Lou, B. Chem. Rev. 2000, 100, 4443.

[10] Jensen, K. J. J. Chem. Soc., Perkin Trans. 1 2002, 2219.

[11] Toshima, K. Carbohydr. Res. 2006, 341, 1282.

[12] Toshima, K.; Sasaki, K. Comprehensive Glycoscience, Vol. 1, Amsterdam, Elsevier, 2007, p. 261.

[13] Matsushita, Y.; Sugamoto, K.; Kita, Y.; Matsui, T. Tetrahedron
Lett. 1997, 38, 8709 .

[14] Chakraborti, A. K.; Gulhane, R. Chem. Commun. 2003, 1896.

[15] Agarwal, A.; Rani, S.; Vankar, Y. D. J. Org. Chem. 2004, 69, 6137.

[16] Misra, A. K.; Tiwari, P.; Agnihotri, G. Synthesis. 2005, 260.

[17] Tiwari, P.; Agnihotriand, G.; Misra, A. K. Carbohydr. Res. 2005, 340, 749.

[18] Colinas, P. A.; Nuńẽz, N. A.; Bravo, R. D. J. Carbohydr. Chem. 2008, 27: 141-147.

[19] Mukhopadhyay, B.; Collet, B.; Field, R. A. Tetrahedron Lett. 2005 , 46, 5923.

[20] Mukherjee, C.; Misra, A. K. Synthesis 2007, 683.

[21] Mukhopadhyay, B.; Maurer, S. V.; Rudolph, N.; van Well, R. M.; Russell, D. A.; Field, R. A. J. Org. Chem. 2005, 70, 9059.

[22] Du, Y.; Wei, G.; Cheng, S.; Hua, Y.; Linhardt, R. J. Tetrahedron Lett. 2006, 47, 307.

[23] Ludek, O. R.; Gu, W.; Gildersleeve, J. C. Carbohydr. Res. 2010, 345, 2074.

[24] Agnihotri, G.; Misra, A. K. Carbohydr. Res. 2006, 341, 2420.

[25] Chun, Y. X.; Yan, S. Q.; Li, X. P.; Ding, N.; Zhang, W.; Wang, P.; Li, Y. X.; Li, M. Tetrahedron Lett. 2011, 52, 6196.

[26] Roy, B.; Mukhopadhyay, B. Tetrahedron Lett. 2007, 48, 3783.

[27] Shaikh, N.; Russo, L.; Cipolla, L.; Nicotra, F. Mol. Diversity 2011, 15,341 .

[28] Richel, A.; Laurent, P.; Wathelet, B.; Wathelet, J. P.; Paquot, M. Tetrahedron Lett. 2010, 51, 1356.

[29] Zhou, J. F.; Chen, X.; Wang, Q. B.; Zhang, B.; Zhang, L. Y.; Yusulf, A.; Wang, Z. F.; Zhang, J. B.; Tang, J. Chin. Chem. Lett. 2010 , 21,922 .

[30] Dasgupta, S.; Pramanik, K.; Mukhopadhyay, B. Tetrahedron 2007, 63, 12310.

[31] Mandaland, S.; Mukhopadhyay, B. Tetrahedron 2007, 63, 11363.

[32] Rajput, V. K.; Mukhopadhyay, B. J. Org. Chem. 2008, 73, 6924.

[33] Roy, B.; Pramanik, K.; Mukhopadhyay, B. Glycoconjugate J. 2008, $25,157$.

[34] Dasgupta, S.; Mukhopadhyay, B. Eur. J. Org. Chem. 2008, 5770.

[35] Roy, B.; Field, R. A.; Mukhopadhyay, B. Carbohydr. Res. 2009, $344,2311$.

[36] Verma, P.; Mukhopadhyay, B. Carbohydr. Res. 2009, 344, 2554.

[37] Wu, Y.; Yu, J. G.; Ma, X. F.; Zhang, J. S. Chin. Chem. Lett. 2007, $18,1173$.

[38] Rodríguez, O. M.; Colinas, P. A.; Bravo, R. D. Synlett 2009, 1154

[39] Kinfe, H. H.; Mebrahtu, F. M.; Sithole, K. Carbohydr. Res. 2011, $346,2528$.

[40] Misra, A. K.; Tiwari, P.; Madhusudan, S. K. Carbohydr. Res. 2005, 340, 325.

[41] Mukhopadhyay, B.; Russell, D. A.; Field, R. A. Carbohydr. Res. 2005, 340, 1075 .

[42] Agarwal, A.; Vankar, Y. D. Carbohydr. Res. 2005, 340, 1661.

[43] Agnihotri, G.; Misra, A. K. Tetrahedron Lett. 2006, 47, 3653.

[44] Agnihotri, G.; Tiwari, P.; Misra, A. K. J. Carbohydr. Chem. 2006, 25,491 .

[45] Liu, H. X.; Wu, Q. P.; Shu, Y. N.; Chen, X.; Xi, X. D.; Du, T. J.; Zhang, Q. S. Carbohydr. Res. 2009, 344, 2342.

[46] Du, T. J.; Wu, Q. P.; Liu, H. X.; Chen, X.; Shu, Y. N.; Xi, X. D.; Zhang, Q. S.; Li, Y. Z. Tetrahedron 2011, 67, 1096.

[47] Wu, H.; Shen, Y.; Fan, L. Y.; Wan, Y.; Shi, D. Q. Tetrahedron 2006, 62, 7995 .

[48] Mukhopadhyay, B. Tetrahedron Lett. 2006, 47, 4337.

[49] Rajput, V. K.; Mukhopadhyay, B. Tetrahedron Lett. 2006, 47, 5939.

[50] Rajput, V. K.; Roy, B.; Mukhopadhyay, B. Tetrahedron Lett. 2006, $47,6987$. 
[51] Roy, B.; Verma, P.; Mukhopadhyay, B. Carbohydr. Res. 2009, 344, 145.

[52] Yan, S. Q.; Ding, N.; Zhang, W.; Wang, P.; Li, Y. X.; Li, M. Carbohydr. Res. 2012, 354, 6.

[53] Kumar, G. D. K.; Baskaran, S. J. Org. Chem. 2005, 70, 4520.

[54] Kumar, P. S.; Kumar, G. D. K.; Baskaran, S. Eur. J. Org. Chem. 2008, 6063.

[55] Yadav, J. S.; Raghavendra, S.; Satyanarayana, M.; Balanarsaiah, E. Synlett 2005, 2461.
[56] Das, B.; Mahender, G.; Kumar, V. S.; Chowdhury, N. Tetrahedron Lett. 2004, 45, 6709.

[57] Niu, Y.; Wang, N.; Cao, X.; Ye, X. S. Synlett 2007, 2116.

[58] Mahender, G.; Ramu, R.; Ramesh, C.; Das, B. Chem. Lett. 2003, 734.

[59] Tiwari, P.; Misra, A. K. Tetrahedron Lett. 2006, 47, 3573.

[60] Dasgupta, S.; Roy, B.; Mukhopadhyay, B. Carbohydr. Res. 2006, 341,2708 . 